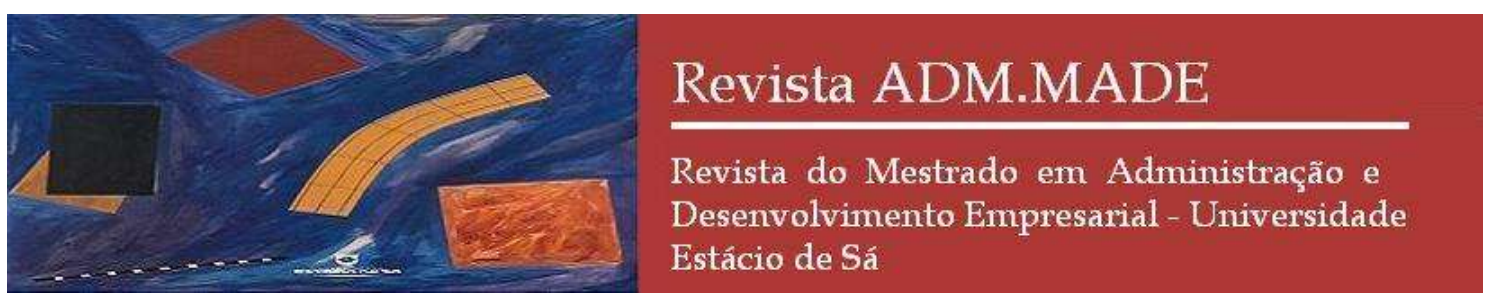

Revista ADM.MADE, Rio de Janeiro, ano 16, v.20, n.2, p.23-40, maio/agosto, 2016

http://dx.doi.org/10.21714/2237-51392016v20n2p023040

Revista do Mestrado em Administração e Desenvolvimento Empresarial da Universidade

Estácio de Sá - Rio de Janeiro (MADE/UNESA). ISSN: 2237-5139

Conteúdo publicado de acesso livre e irrestrito, sob licença Creative Commons 3.0.

Editor responsável: Marco Aurélio Carino Bouzada

\title{
Benefícios e Riscos na Avaliação de Projetos de Pesquisa e de Desenvolvimento no Setor Elétrico Brasileiro
}

\author{
José Roberto Ribas ${ }^{1}$ \\ Luciana Fernandes Guimarães ${ }^{2}$ \\ Camila Moura Caiaffa ${ }^{3}$
}

\footnotetext{
Artigo recebido em 12/11/2015 e aprovado em 02/03/2016. Artigo avaliado em double blind review.

${ }^{1}$ Doutor em Administração pela Escola de Administração de Empresas de São Paulo, Fundação Getúlio Vargas (EAESP/FGV). Professor Adjunto IV da Escola Politécnica da Universidade Federal do Rio de Janeiro. Endereço: Av. Athos da Silveira Ramos, 149 - Prédio do Centro de Tecnologia - Bloco F / 1 andar - Ilha do Fundão - CEP: 21941-909 - Rio de Janeiro, RJ. Email: ribas@poli.ufrj.br.

${ }^{2}$ Mestre em Engenharia Civil pelo Instituto Alberto Luiz Coimbra de Pós-Graduação e Pesquisa de Engenharia, Universidade Federal do Rio de Janeiro (COPPE/UFRJ). Pesquisadora pela Fundação para o Desenvolvimento de Bauru (FUNDEB). Endereço: Rua Luis Belart, 101/301 - Ilha do Governador - CEP: 21941-100 - Rio de Janeiro, RJ. Email: lucianafg@poli.ufrj.br.

${ }^{3}$ Mestre em Logística pela PUC-RIO, Engenheira da Assessoria de Projetos Especiais da Light Serviços de Eletricidade S/A. Av. Marechal Floriano, 168 - Bloco 4ㅇ - 2o andar - CEP: 20080-002 - Rio de Janeiro, RJ. Email: camilacaiaffa@hotmail.com.
} 


\section{Benefícios e Riscos na Avaliação de Projetos de Pesquisa e de Desenvolvimento no Setor Elétrico Brasileiro}

A presente pesquisa tem, por objetivo, propor um procedimento metodológico para a classificação de projetos de pesquisa e desenvolvimento (P\&D) no setor elétrico brasileiro relativamente a dois aspectos: o benefício e o número de prioridade de risco (NPR). O primeiro representa uma agregação de valor enquanto o segundo representa o risco associado. Os pesos para ambas as medidas são obtidos por meio da técnica multicritério Fuzzy Analytic Hierarchy Process (FAHP). O desempenho que cada projeto apresenta relativamente aos benefícios e aos riscos é mensurado por meio de uma escala ordinal de importância para os benefícios e de probabilidade de ocorrência versus impacto das consequências para os riscos. Ao final, os projetos são posicionados em um gráfico com eixos ortogonais, possibilitando análises absolutas e relativas. É apresentado um estudo de caso envolvendo a identificação de seis benefícios e seis eventos de risco aplicados a cinco projetos de P\&D, com o intuito de demonstrar e de validar a metodologia proposta.

Palavras-chave: Projetos de P\&D, Benefícios, Direcionadores de Risco, Números de Prioridade de Risco, Fuzzy AHP

Keywords: R\&D Projects, Benefits, Risk Drivers, Risk Priority Numbers, Fuzzy AHP.

\section{Benefits and Risks Assessment for the Brazilian Electric Industry R\&D Projects}

The present research aims at proposing a methodological procedure to rank Research and Development (R\&D) projects in the Brazilian electric industry in respect of two issues: their benefit and risk priority number (RPN). The former means the value added and the later means the related risk. The weights of both measures are achieved through a multicriteria technique called Fuzzy Analytic Hierarchy Process (FAHP). The performance of each project related to their benefits and risks are assessed by means of an ordinal scale of importance for benefits and the likelihood of occurrence versus the consequence impacts for risks. At the end, the projects are scattered in an orthogonal chart, making it possible to perform absolute and relative evaluation. Aiming at demonstrating and validating the proposed methodology, a case study was presented comprising the identification of six benefits and six risk events applied to five R\&D projects is presented.

\section{INTRODUÇÃO}

A legislação brasileira determina que as empresas que atuam no mercado de energia elétrica no Brasil devem aplicar uma parcela de sua receita operacional líquida em pesquisa e desenvolvimento (P\&D) tecnológico do setor de energia elétrica. São isentas dessa obrigação apenas as empresas autorizadas de produção independente que geram energia a partir de instalações eólica, solar ou de biomassa, ou de cogeração qualificada, ou pequenas centrais hidroelétricas. Conforme disposto no art. 40 da Lei $n$ ㅇ 9.991/2000, os investimentos em P\&D devem ser distribuídos do seguinte modo: (i) 40\% devem ser recolhidos ao Fundo Nacional de Desenvolvimento Científico e Tecnológico (FNDCT); (ii) $40 \%$ devem ser destinados à execução de projetos de P\&D regulados pela ANEEL; (iii) $20 \%$ devem ser recolhidos ao Ministério de Minas e Energia (MME).

Segundo regulamentos estabelecidos pela ANEEL, esses projetos devem ser "destinados à capacitação e ao desenvolvimento tecnológico das empresas de energia 
elétrica, visando à geração de novos processos ou produtos, ou o aprimoramento de suas características" (ANEEL, 2012, p.14). Os projetos podem ser desenvolvidos independente ou cooperativamente entre duas ou mais empresas, com instituições de ensino ou de pesquisa, com empresas de consultoria e com fabricantes de materiais e de equipamentos.

Para que os projetos de uma empresa sejam válidos como investimentos em P\&D, é necessário enviar à ANEEL o escopo dos projetos, com informações a respeito dos resultados esperados, da sua aplicabilidade, dos custos previstos para sua execução, da expectativa de retorno financeiro, da pertinência do estudo a temas de interesse do setor elétrico, e do grau de inovação ou de avanço tecnológico pretendido.

Após seu encerramento, o projeto passa por uma avaliação dos resultados alcançados e dos gastos incorridos, para fins de aprovação do projeto e de reconhecimento dos investimentos realizados pela ANEEL. A análise dos resultados do projeto leva em conta os critérios originalidade, aplicabilidade, relevância e razoabilidade dos custos. A cada critério é atribuída uma pontuação que determinará a nota do projeto, a qual definirá sua aprovação, que pode ser total ou parcial, ou sua reprovação. No caso de projetos parcialmente aprovados ou reprovados, os gastos não reconhecidos devem ser estornados à conta de P\&D e corrigidos pela taxa do Sistema Especial de Liquidação e de Custódia (SELIC).

De modo a garantir que seus projetos estejam adequados aos requisitos da ANEEL e sejam relevantes para as concessionárias e para seus stakeholders, as primeiras selecionam projetos de pesquisa que sejam de interesse e, ao mesmo tempo, tenham chance razoável de obter aprovação total pela ANEEL.

Esse artigo propõe uma metodologia para identificar o benefício consolidado para a empresa executora e o risco agregado na forma do número de prioridade de risco (NPR) para cada projeto candidato a compor a carteira de projetos de P\&D de uma instituição, com o propósito de permitir avaliar cada um deles sob a ótica do risco versus o retorno.

\section{REFERENCIAL TEÓRICO}

\subsection{Risco em Projetos de Pesquisa e Desenvolvimento}

Um risco é qualquer evento que pode afetar, positiva ou negativamente, os objetivos de um projeto. Qualquer obra de engenharia está sujeita a riscos e a incertezas. A escala de uma usina hidrelétrica está diretamente relacionada ao tamanho do impacto que um evento inesperado possa vir a causar, entretanto, não se limita apenas a isso.

A complexidade, o cronograma e a localização, dentre outros, também são fatores que interferem no perfil de risco. A usina está exposta a riscos desde o início da sua construção até a entrada em operação, sendo sua natureza e intensidade dinâmica vinculada ao estágio da obra e a eventos que, de modo combinado, podem vir a desencadear processo com resultados não planejados. Alguns desses eventos são incontroláveis e externos, muito vinculados ao clima e às condições geológicas. Outros são parcial ou totalmente controláveis, podem ser mitigados ou até mesmo evitados, a exemplo da segurança dos trabalhadores. 
A identificação dos riscos se concentra naqueles que podem afetar o sucesso do projeto, e busca verificar suas características (TEOH; CASE, 2004). Trata-se de um processo iterativo, visto que outros podem ser descobertos durante todo o ciclo de vida do projeto. Dessa forma, a idéia principal do processo de identificação é determinar e analisar, de maneira preventiva, os fatores relevantes que poderiam acontecer e que apresentariam um impacto importante no alcance dos objetivos do projeto. Esse processo de identificação, quando bem feito, pode reduzir futuros custos e surpresas indesejadas ao longo da construção. Quanto mais tarde - ao longo da linha temporal do projeto - um dado risco for identificado, maiores serão os custos a que o empreendedor estará sujeito na implantação das medidas para sua mitigação.

A metodologia multicritério tem sido utilizada com sucesso na identificação e na análise de risco em projetos de inovação tecnológica (FIGUEIRA; GRECO; EHRGOTT, 2005) e sua utilização nas fases iniciais do projeto tem proporcionado resultados úteis. O uso de conjuntos fuzzy associado a técnicas multicritério pode ser observado em projetos de pesquisa governamentais (HUANG; CHU; CHIANG, 2008) e tem se revelado de grande utilidade (PAN, 2008; ZAVADSKAS et al., 2008).

\subsection{Fuzzy Analytic Hierarchy Process (FAHP)}

Para se decidir sobre qual método dentre os disponíveis para análise de decisão multicritério, ou na língua inglesa Multiple-Criteria Decision Analysis (MCDA), é mais adequado para a análise a ser desenvolvida na presente pesquisa, deve-se considerar que, quando as informações do processo decisório são baseadas em opiniões do decisor de um programa com relação aos critérios de escolha, há uma preocupação quanto às imprecisões e às ambiguidades dos processos de decisão.

O FAHP (CHANG, 1996) surge como uma solução para esse tipo de processo decisório, quando imprecisões dificultam uma tomada de decisão correta (TANG; BEYNON, 2005), tornando-o, portanto, mais eficiente que o método AHP. Ademais, sua vantagem sobre os métodos de sobreclassificação como o Promethee e o Electre é de que o trabalho de especificação do modelo é mais transparente, com a ressalva de que todos os elementos sejam comparáveis entre si (MONTIS et al., 2005).

O método a ser utilizado em casos desse tipo deve agregar, de maneira satisfatória, as avaliações dos diversos participantes do processo de decisão, bem como as imprecisões inerentes as essas avaliações (MEIXNER, 2011). A utilização do método AHP é enfraquecida quando são levadas em consideração as imprecisões existentes nas decisões.

Um determinado número fuzzy é caracterizado por uma função de pertinência $\mu_{A}(x)$ que, por sua vez, assume valores no intervalo $\{0,1\}$. Os números fuzzy triangulares são bastante utilizados, uma vez que têm simplicidade computacional que facilita o tratamento de dados (TANG; BEYNON, 2005).

Seja $A=\left\{a_{i j}\right\}_{n \times n}$ um conjunto de comparações pareadas entre critérios ou modalidades, de tal modo que $a_{i j}=\left\{I_{i j}, m_{i j}, u_{i j}\right\}$ para uma função de pertinência triangular, representando os valores mínimo, médio e máximo, respectivamente. 
Para as comparações pareadas reversas, supondo transitividade, $a_{j i}=\left\{\frac{1}{l_{i j}}, \frac{1}{m_{i j}} \frac{1}{u_{i j}}\right\}$.

Seja ainda um conjunto de números fuzzy pertinentes às comparações pareadas $a_{i j}$, conforme uma função triangular. Então a medida sintética fuzzy $(S)$ para cada $a_{i j}$ é determinada por:

$$
\mathrm{S}_{\mathrm{i}}=\sum_{\mathrm{j}=1}^{\mathrm{n}} \mathrm{a}_{\mathrm{ij}} \odot\left[\sum_{\mathrm{i}=1}^{\mathrm{n}} \sum_{\mathrm{j}=1}^{\mathrm{n}} \mathrm{a}_{\mathrm{ij}}\right]^{-1}
$$

Sendo $S_{i}=\left\{l_{i}, m_{i}, u_{i}\right\}$.

Com as medidas sintéticas fuzzy $(S)$ calculadas, fazem-se as comparações entre os $S$ de cada um dos benefícios ou modalidades, por meio do cálculo de $V\left(S_{i} \geq S_{j}\right)$. Deve-se realizar esse cálculo dois a dois, entre todos os pares de benefícios ou modalidades possíveis.

Para verificar dois números fuzzy triangulares convexos, conforme a Figura 1 , a exemplo de $S_{1}$ e $S_{2}$, números esses que se interceptam, a equação (2) deve ser utilizada, na qual $V$ representa a probabilidade de $M_{1} \geq M_{2}$ (CHANG, 1996):

$$
\begin{aligned}
& V\left(S_{1} \geq S_{2}\right)=1 \text { se e somente se } m_{1} \geq m_{2} \\
& V\left(S_{2} \geq S_{1}\right)=\operatorname{máx}\left(S_{1} \cap S_{2}\right)=\left(I_{1}-u_{2}\right) /\left[\left(m_{2}-u_{2}\right)-\left(m_{1}-l_{1}\right)\right]
\end{aligned}
$$

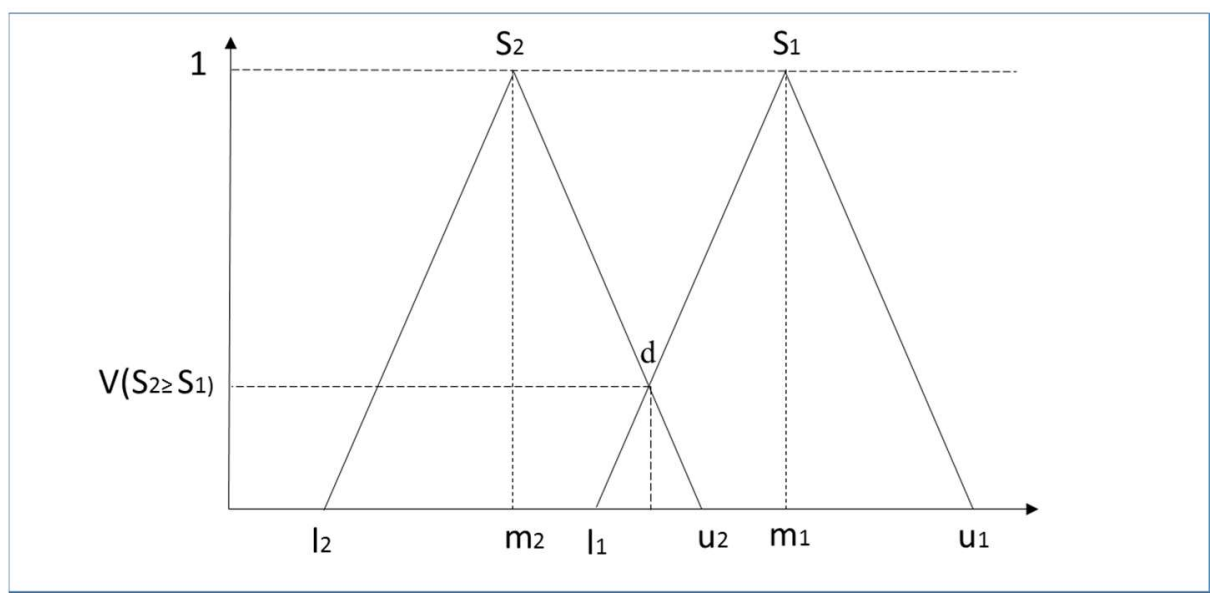

Figura 1 - Comparação entre dois números fuzzy (CHANG, 1996, p.651)

Supondo que tenhamos cinco benefícios, para determinar a probabilidade $d\left(C_{1}\right)$ de que o primeiro benefício " 1 " seja superior aos demais, procedimento este válido também para os riscos, comparam-se as medidas sintéticas fuzzy de $S_{1}$ com as demais $S_{2}, S_{3}, S_{4}$ e $S_{5}$, conforme as equações [2] e [3], e adota-se o menor valor obtido destas comparações conforme a equação [4].

$$
\mathrm{d}\left(C_{1}\right)=V\left(S_{1} \geq S_{2}, S_{3}, S_{4}, S_{5}\right)=\operatorname{Min}\left(V\left(S_{1} \geq S_{2}\right), V\left(S_{1} \geq S_{3}\right), V\left(S_{1} \geq S_{4}\right), V\left(S_{1} \geq S_{5}\right)\right)
$$

O vetor $W$, definido como $W=[d(C 1), d(C 2), d(C 3), d(C 4), d(C 5)]$, após ser normalizado, será o vetor das ordens de importância dos benefícios. 


\section{DESENVOLVIMENTO DA METODOLOGIA}

O procedimento consiste em três fases: a) identificação e cadastro das entidades; b) estimativa dos pesos dos benefícios e dos direcionadores de risco; c) estimativa dos desempenhos do projeto. As três fases são encadeadas, ou seja, será possível executar "2" apenas quando "1" tiver, no mínimo, dois benefícios e dois direcionadores de risco cadastrados. A execução de " 3 " depende de: i) que, em " 1 ", tenha sido cadastrado pelo menos um projeto; ii) "2" tenha sido executada. O intervalo de tempo entre as fases é variável e a atualização do cadastro de entidades pode ocorrer a qualquer momento (RIBAS, 2015).

\subsection{Fase "1" - Identificação e cadastro das entidades}

Os benefícios e os direcionadores de risco identificados são cadastrados com código e com identificação. Os projetos, por sua vez, possuem código, identificação, ano de início, duração e tipo de solução (ambiental, automação). A manutenção, significando inclusão, exclusão ou alteração, do banco de dados contendo essas três entidades ocorre de modo independente.

Visando a demonstrar a funcionalidade da metodologia proposta, optou-se por selecionar 12 critérios utilizados atualmente no processo de apreciação das propostas e de projetos (LIGHT, 2014), informação secundária que abrevia o tempo dispendido na condução das entrevistas. O Quadro 1 apresenta a lista de benefícios, e o Quadro 2 a lista de direcionadores de risco selecionados para especificar o presente estudo.

Quadro 1 - Relação dos Benefícios Esperados dos Projetos de P\&D

\begin{tabular}{c|l}
\hline \hline Código & Título \\
\hline B1 & $\begin{array}{l}\text { Sustentabilidade (apelo social, ambiental e } \\
\text { financeiro) }\end{array}$ \\
\hline B2 & Solução tecnológica e facilidade de internalização \\
\hline B3 & Potencial de comercialização \\
\hline B4 & $\begin{array}{l}\text { Publicação em anais de congresso e periódicos } \\
\text { acadêmicos }\end{array}$ \\
\hline B5 & Capacitação de pessoal da empresa patrocinadora \\
\hline B6 & Obtenção de patente ou de registro de software \\
\hline \hline
\end{tabular}

Fonte: Elaborado pelos autores. 
Quadro 2 - Relação dos Direcionadores de Risco dos Projetos de P\&D

\begin{tabular}{c|l}
\hline \hline Código & Título \\
\hline R1 & Caráter de inovação tecnológica \\
\hline R2 & Desempenho pregresso do executor \\
\hline R3 & $\begin{array}{l}\text { Adequação da capacitação e disponibilidade da equipe } \\
\text { executora }\end{array}$ \\
\hline R4 & $\begin{array}{l}\text { Comprometimento do valor do projeto no orçamento } \\
\text { de P\&D }\end{array}$ \\
\hline R5 & Prazo adequado para o escopo pretendido \\
\hline R6 & $\begin{array}{l}\text { Adequação dos recursos materiais, equipamentos e } \\
\text { despesas }\end{array}$ \\
\hline \hline
\end{tabular}

Fonte: Elaborado pelos autores.

\subsection{Fase "2a" - Estimativa dos Pesos dos Benefícios}

O método Simos (FIGUEIRA; ROY, 2002) para a manifestação das preferências de um decisor atua de forma objetiva nessa tarefa, pois preenche uma característica na comparação pareada que é desfavorável. Isso porque, conforme proposta por Saaty (SAATY, 1980), as avaliações devem ser realizadas duas a duas entre todos os elementos comparáveis. Esse requisito demanda trabalho considerável no exercício de avaliações quando o número de elementos é grande. Nesse aspecto em particular, o método Simos é mais rápido, uma vez que o encadeamento é sequencial.

Adotando este método, foram apresentadas, ao especialista de P\&D, as seis cartas identificadoras, semelhantes a um baralho, contendo os títulos dos benefícios, constantes no Quadro 1. O especialista tinha também à sua disposição um total de 18 cartas em branco.

A primeira orientação estabelecida foi a seguinte: "Observando este conjunto de benefícios que a empresa espera de um projeto de inovação, qual dentre eles você acredita ser o de maior importância?".

Uma vez selecionada a carta identificadora, a pergunta seguinte foi "Seguindo este mesmo princípio, qual seria o segundo em ordem de importância?".

Tendo sido selecionadas as duas cartas identificadoras, a pergunta seguinte foi "Determine agora o grau de importância que o primeiro possui relativamente ao segundo. Uma carta branca entre os dois significará que um é pouco mais importante que outro, duas cartas brancas significarão que um é mais importante que outro, três cartas em branco significarão que um é muito mais importante que outro".

Determinado o grau de importância, o processo prosseguia com a seleção da carta identificadora seguinte. Quando não existia preferência entre dois benefícios - ou seja, eram igualmente importantes - não ocorria separação com cartas em branco. 
A Tabela 1 mostra a ordem de preferência na primeira coluna, a sequência ordinal de todas as cartas incluindo as brancas na segunda coluna, a sequência ordinal apenas das cartas identificadoras na terceira coluna e a posição média dos benefícios na quarta coluna. Para a escala de Saaty (1980; 2009), a posição média é convertida para a base 8 conforme pode ser observada na quinta coluna e, em seguida, calculada a pontuação de cada benefício verificada na sexta coluna (RIBAS, 2015).

Tabela 1 - Escores de Importância dos Benefícios

\begin{tabular}{c|c|c|c|c|c}
\hline \multirow{2}{*}{ Ordem } & \multicolumn{3}{|c|}{ Simos } & \multicolumn{2}{c}{ Escala Saaty } \\
\cline { 2 - 6 } & Sequência & Posição & Média & Base 8 & Pontuação \\
\hline B6 & 1 & 1 & 1,0 & 2,1 & 2 \\
\hline branca & 2 & - & - & - & - \\
\hline B4 & 3 & 2 & 2,0 & 3,1 & 3 \\
\hline branca & 4 & - & - & - & - \\
\hline branca & 5 & - & - & - & - \\
\hline B3 & 6 & 4 & 4,0 & 5,3 & 5 \\
\hline branca & 7 & - & - & - & - \\
\hline branca & 8 & - & - & - & - \\
\hline B5 & 9 & 6 & 6,0 & 7,4 & 7 \\
\hline branca & 10 & - & - & - & - \\
\hline B2 & 11 & 7 & 7,5 & 9,0 & 9 \\
\hline B1 & 12 & 8 & 7,5 & 9,0 & 9 \\
\hline \hline
\end{tabular}

Fonte: Elaborado pelos autores.

A pontuação dos benefícios representada na última coluna da Tabela 1 é utilizada para compor a matriz de importâncias relativas da Tabela 2, observando que a diagonal principal é igual a 1 e que as células abaixo da diagonal representam os valores inversos das suas posições simétricas superiores.

Tabela 2 - Graus de Importância dos Benefícios na Escala de Saaty (1980; 2009)

\begin{tabular}{|c|c|c|c|c|c|c|}
\hline & B1 & B2 & B5 & B3 & B4 & B6 \\
\hline B1 & 1 & 1 & 3 & 5 & 7 & 8 \\
\hline B2 & 1 & 1 & 3 & 5 & 7 & 8 \\
\hline B5 & $1 / 3$ & $1 / 3$ & 1 & 3 & 5 & 6 \\
\hline
\end{tabular}




\begin{tabular}{c|cccccc} 
B3 & $1 / 5$ & $1 / 5$ & $1 / 3$ & 1 & 3 & 4 \\
B4 & $1 / 7$ & $1 / 7$ & $1 / 5$ & $1 / 3$ & 1 & 2 \\
B6 & $1 / 8$ & $1 / 8$ & $1 / 6$ & $1 / 4$ & $1 / 2$ & 1 \\
\hline \hline
\end{tabular}

Fonte: Elaborado pelos autores.

O procedimento Fuzzy AHP (CHANG, 1996; ZHUA; JINGA; CHANG, 1999; LEUNGA; CAOB, 2000) é aplicado a partir de dois elementos de entrada; a matriz da Tabela 2 traduz os graus de importância entre os pares de benefícios; e o grau de fuzzificação indica o deslocamento nestes valores, considerados como modais ou crisp ${ }^{4}$.

Dadas as características subjetivas da priorização pelo método Simos, optou-se por adotar valor igual a 3, deslocamento esse capaz de inverter as preferências entre dois elementos quando um deles é um pouco mais importante que o outro. Os resultados para os pesos não normalizados dos benefícios após a aplicação do método estão exibidos na Tabela 3. Eles refletem, em última análise, o grau de importância relativo que cada qual possui quando comparado aos demais.

Tabela 3 - Vetor de Pesos para os Benefícios

\begin{tabular}{c|c|c|c|c|c}
\hline \hline B1 & B2 & B3 & B4 & B5 & B6 \\
\hline 1,00000 & 1,00000 & 0,64263 & 0,39198 & 0,93041 & 0,23494 \\
\hline \hline
\end{tabular}

Fonte: Elaborado pelos autores.

Verifica-se que "sustentabilidade (apelo social, ambiental e financeiro)" e "solução tecnológica e facilidade de internalização" seguidos por "potencial de comercialização" são julgados pelo especialista em P\&D como os resultados mais importantes que ele espera de um projeto de P\&D.

\subsection{Fase "2b" - Estimativa dos Pesos dos Direcionadores de Risco}

Do mesmo modo, as seis cartas identificadoras dos direcionadores de risco e outras 18 cartas em branco foram apresentadas ao especialista de P\&D contendo seus títulos conforme o Quadro 2. A primeira orientação estabelecida foi a seguinte: "Observando esse conjunto de direcionadores de risco que ameaçam os resultados esperados para um projeto de inovação, qual dentre eles você acredita ser aquele que mais compromete tais resultados?".

Uma vez selecionada a carta identificadora, a pergunta seguinte era "Seguindo este mesmo princípio, qual seria o segundo em ordem de importância?".

Tendo sido selecionadas as duas cartas identificadoras, a pergunta seguinte foi "Determine agora o grau de prioridade relativo a ser dedicado na mitigação que o primeiro

\footnotetext{
${ }^{4}$ A denominação crisp é utilizada para indicar valores não fuzzificados. Portanto, representa os resultados modais decorrentes de um grau de fuzzificação $(\delta)$ igual a zero.
}

Revista ADM.MADE, Rio de Janeiro, ano 16, v.20, n.2, p.23-40, maio/agosto, 2016. 
apresenta relativamente ao segundo. Uma carta branca entre os dois significará que a prioridade de um é pouco maior que a do outro, duas cartas brancas significarão que a prioridade de um é maior que a do outro, três cartas em branco significarão que a prioridade de um é muito maior que a do outro".

Determinado o grau de prioridade, o processo prosseguia com a seleção da carta identificadora seguinte. Da mesma forma, quando não existia preferência entre dois direcionadores de risco, por ambos possuírem igual prioridade, não ocorria separação com cartas em branco.

A Tabela 4 mostra a ordem de preferência para os riscos cuja apresentação é semelhante aos valores apresentados na Tabela 1 para os benefícios.

Tabela 4 - Graus de Prioridade entre Direcionadores de Risco

\begin{tabular}{c|c|c|c|c|c}
\hline \multirow{2}{*}{ Ordem } & \multicolumn{3}{|c|}{ Simos } & \multicolumn{2}{c}{ Escala Saaty } \\
\cline { 2 - 6 } & Sequência & Posição & Média & Base 8 & Pontuação \\
\hline R4 & 1 & 1 & 1 & 1,8 & 2 \\
\hline branca & 2 & - & - & - & - \\
\hline branca & 3 & - & - & - & - \\
\hline R5 & 4 & 3 & 3 & 3,4 & 3 \\
\hline branca & 5 & - & - & - & - \\
\hline branca & 6 & - & - & - & - \\
\hline R2 & 7 & 5 & 5 & 5,0 & 5 \\
\hline branca & 8 & - & - & - & - \\
\hline R3 & 9 & 6 & 6 & 5,8 & 6 \\
\hline branca & 10 & - & - & - & - \\
\hline R6 & 11 & 7 & 7 & 6,6 & 7 \\
\hline branca & 12 & - & - & - & - \\
\hline branca & 13 & - & - & - & - \\
\hline branca & 14 & - & - & - & 9 \\
\hline R1 & 15 & 10 & 10 & 9,0 & - \\
\hline
\end{tabular}

Fonte: Elaborado pelos autores.

A pontuação dos direcionadores de risco pela escala Saaty apresentada na última coluna da etapa 3 é aquela cujas diferenças relativas formam a Tabela 5. 
Tabela 5 - Graus de Importância dos Direcionadores de Risco na Escala de Saaty

\begin{tabular}{c|cccccc}
\hline \hline & $\mathrm{R} 1$ & $\mathrm{R} 6$ & $\mathrm{R} 3$ & $\mathrm{R} 2$ & $\mathrm{R} 5$ & $\mathrm{R} 4$ \\
\hline $\mathrm{R} 1$ & 1 & 3 & 4 & 5 & 7 & 8 \\
$\mathrm{R} 6$ & $1 / 3$ & 1 & 2 & 3 & 5 & 6 \\
$\mathrm{R} 3$ & $1 / 4$ & $1 / 2$ & 1 & 2 & 4 & 5 \\
$\mathrm{R} 2$ & $1 / 5$ & $1 / 3$ & $1 / 2$ & 1 & 3 & 4 \\
$\mathrm{R} 5$ & $1 / 7$ & $1 / 5$ & $1 / 4$ & $1 / 3$ & 1 & 2 \\
$\mathrm{R} 4$ & $1 / 8$ & $1 / 6$ & $1 / 5$ & $1 / 4$ & $1 / 2$ & 1 \\
\hline \hline
\end{tabular}

Fonte: Elaborado pelos autores.

O procedimento Fuzzy AHP (ZHUA; JINGA; CHANG, 1999) é também aplicado a partir da matriz da Tabela 5 e do grau de fuzzificação igual a 3. Os resultados para os pesos não normalizados dos direcionadores de risco encontram-se na Tabela 3. Estes valores são estimativas dos números de prioridades de risco (NPR), e refletem o produto entre a probabilidade e o impacto das consequências.

Tabela 6 - Vetor de Pesos para os Direcionadores de Risco

\begin{tabular}{c|c|c|c|c|c}
\hline \hline R1 & R2 & R3 & R4 & R5 & R6 \\
\hline 1,00000 & 0,63696 & 0,75426 & 0,22146 & 0,41596 & 0,85195 \\
\hline \hline
\end{tabular}

Fonte: Elaborado pelos autores.

O "caráter de inovação tecnológica", seguido da "adequação dos recursos materiais, equipamentos e despesas" são os direcionadores com maior NPR, e, portanto, aqueles que devem ser preferencialmente atendidos de maneira satisfatória pelo projetos - ou, na impossibilidade, mitigados pelos gestores de P\&D.

\subsection{Fase "3a" - Relação dos Projetos Avaliados}

Os cinco projetos que serão avaliados quanto aos benefícios que proporcionam e quanto aos riscos a que estão sujeitos estão descritos no Quadro 3. 
Quadro 3 - Relação de Projetos Avaliados

\begin{tabular}{|c|c|c|}
\hline Código & Título & Descrição \\
\hline P1 & $\begin{array}{l}\text { Otimização de } \\
\text { investimentos em } \\
\text { novas tecnologias de } \\
\text { perdas }\end{array}$ & $\begin{array}{l}\text { Sistema de clusterização geoelétrica por } \\
\text { retorno econômico a cada tipo de } \\
\text { investimento de redução de perdas (ações } \\
\text { convencionais, blindagem de rede e novas } \\
\text { tecnologias - smart meters) e de otimização } \\
\text { por modelagem matemática para } \\
\text { priorização dos investimentos }\end{array}$ \\
\hline P2 & $\begin{array}{c}\text { Modelo de Inteligência } \\
\text { Estratégica }\end{array}$ & $\begin{array}{l}\text { Desenvolvimento de um modelo integrado } \\
\text { de inteligência, risco e inovação apoiando a } \\
\text { alta direção da empresa nos seus processos } \\
\text { decisórios, minimizando riscos e } \\
\text { alavancando oportunidades. }\end{array}$ \\
\hline P3 & $\begin{array}{l}\text { Compensação de } \\
\text { Potência Reativa } \\
\text { Distribuída em Baixa } \\
\text { Tensão }\end{array}$ & $\begin{array}{l}\text { Metodologia para a compensação reativa } \\
\text { na rede de distribuição de baixa tensão, } \\
\text { considerando aspectos como padrões de } \\
\text { consumo e suporte técnico às principais } \\
\text { classes de consumidores. }\end{array}$ \\
\hline P4 & $\begin{array}{l}\text { Gerenciamento de } \\
\text { Identidade e } \\
\text { Autenticação Única }\end{array}$ & $\begin{array}{l}\text { Desenvolvimento de um portal de } \\
\text { autoatendimento para os usuários, com } \\
\text { sincronismo de senha entre os sistemas } \\
\text { implementados, integração e correlação } \\
\text { dos logs do ambiente de gerenciamento de } \\
\text { identidade e SAP, para combate a fraudes } \\
\text { internas }\end{array}$ \\
\hline P5 & $\begin{array}{l}\text { Qualidade da Água, } \\
\text { Biomanipulação, } \\
\text { Sentinelas Ambientais } \\
\text { em Reservatórios }\end{array}$ & $\begin{array}{l}\text { Ações em reservatórios de aprimoramento } \\
\text { da avaliação da qualidade de água; } \\
\text { avaliação dos estoques existentes de } \\
\text { nutrientes; prognóstico ambiental; manejo } \\
\text { ambiental através da biomanipulação; } \\
\text { sentinelas ambientais. }\end{array}$ \\
\hline
\end{tabular}

Fonte: Elaborado pelos autores.

\subsection{Fase "3b" - Desempenho dos Projetos quanto aos Benefícios}

A importância que os benefícios representam para cada projeto é medida por meio de desempenhos (ZAVADSKAS et al., 2008). No aspecto dos benefícios, os níveis a serem considerados são: 0 - não é aplicável; 1 - abaixo do esperado; 2 - atende ao esperado; 3 supera o esperado. 
A Tabela 7 contém as estimativas subjetivas para cada um dos cinco projetos, manifestadas pelo especialista de $P \& D$, relativas aos seis benefícios. O projeto $P 2$, por exemplo, proporcionará benefícios de sustentabilidade à empresa patrocinadora dentro do esperado.

Tabela 7 - Desempenhos dos Projetos relativos aos Benefícios

\begin{tabular}{|c|c|c|c|c|c|c|}
\hline Código & Título & $\mathrm{P} 1$ & $\mathrm{P} 2$ & P3 & P4 & P5 \\
\hline B1 & $\begin{array}{l}\text { Sustentabilidade (apelo social, ambiental e } \\
\text { financeiro) }\end{array}$ & 3 & 2 & 2 & 0 & 3 \\
\hline B2 & $\begin{array}{l}\text { Solução tecnológica e facilidade de } \\
\text { internalização }\end{array}$ & 2 & 2 & 2 & 1 & 2 \\
\hline B3 & Potencial de comercialização & 2 & 2 & 2 & 1 & 1 \\
\hline B4 & $\begin{array}{l}\text { Publicação em anais e periódicos } \\
\text { acadêmicos }\end{array}$ & 2 & 2 & 2 & 1 & 2 \\
\hline B5 & $\begin{array}{l}\text { Capacitação de pessoal da empresa } \\
\text { patrocinadora }\end{array}$ & 1 & 2 & 2 & 1 & 1 \\
\hline B6 & $\begin{array}{l}\text { Obtenção de patente ou de registro de } \\
\text { software }\end{array}$ & 2 & 1 & 1 & 1 & 2 \\
\hline
\end{tabular}

Fonte: Elaborado pelos autores.

O valor do benefício consolidado para cada projeto é obtido por meio da soma dos produtos do vetor de pesos para os benefícios da Tabela 3 e dos desempenhos dos projetos relativos aos benefícios da Tabela 7. O valor de referência é resultado da mesma soma de produtos. Esse valor pode ser estabelecido ad hoc ou, no presente estudo, representado pelo escore médio dentre aqueles atribuídos pelo especialista, no caso igual a 1,67. A Tabela 8 contém os valores dos benefícios consolidados por projeto.

Tabela 8 - Valor dos Benefícios Consolidados por Projeto e Referência

\begin{tabular}{c|c|c|c|c|c}
\hline \hline P1 & P2 & P3 & P4 & P5 & Ref. \\
\hline 8,47 & 8,16 & 8,16 & 3,20 & 7,83 & 7,00 \\
\hline \hline
\end{tabular}

Fonte: Elaborado pelos autores.

\subsection{Fase " $3 c$ " - Desempenho dos Projetos quanto aos Direcionadores de Risco}

Quanto aos direcionadores de risco, os níveis são: 0 - não aplicável; 1 - probabilidade baixa e impacto baixo; 2 - probabilidade baixa e impacto alto; 2 - probabilidade alta e impacto baixo; 3 - probabilidade alta e impacto alto. 
A Tabela 9 exibe as expectativas do especialista de P\&D para cada um dos cinco projetos, para os seis direcionadores de risco. O projeto P1, por exemplo, está sujeito a uma alta probabilidade de que os objetivos de inovação tecnológica não venham a ser atendidos, assim como o impacto decorrente das consequências do não cumprimento desse requisito é considerado elevado.

Tabela 9 - Desempenhos dos Projetos relativos aos Direcionadores de Risco

\begin{tabular}{|c|c|c|c|c|c|c|}
\hline Código & Título & P1 & $\mathrm{P} 2$ & P3 & P4 & P5 \\
\hline R1 & Caráter de inovação tecnológica & 3 & 1 & 1 & 3 & 1 \\
\hline $\mathrm{R} 2$ & Desempenho pregresso do executor & 3 & 1 & 1 & 3 & 1 \\
\hline R3 & $\begin{array}{l}\text { Adequação da capacitação e disponibilidade } \\
\text { da equipe executora }\end{array}$ & 1 & 1 & 1 & 1 & 1 \\
\hline R4 & $\begin{array}{l}\text { Comprometimento do valor do projeto no } \\
\text { orçamento de P\&D }\end{array}$ & 3 & 1 & 1 & 3 & 1 \\
\hline R5 & Prazo adequado para o escopo pretendido & 3 & 1 & 1 & 3 & 1 \\
\hline R6 & $\begin{array}{l}\text { Adequação dos recursos materiais, } \\
\text { equipamentos e despesas }\end{array}$ & 1 & 1 & 1 & 1 & 1 \\
\hline
\end{tabular}

Fonte: Elaborado pelos autores.

O número de prioridade de risco para cada projeto é obtido por meio da soma dos produtos do vetor de pesos para os direcionadores de risco da Tabela 6 e os desempenhos dos projetos relativos aos riscos da Tabela 9. Assim como no cálculo dos benefícios, o valor de referência é resultado da mesma soma de produtos, por julgamento ad hoc ou, também aqui, obtido pelo escore médio dentre aqueles atribuídos pelo especialista, no caso igual a 1,53. A Tabela 10 apresenta os valores por projeto.

Tabela 10 - Números de Prioridade de Risco por Projeto e Referência

\begin{tabular}{c|c|c|c|c|c}
\hline \hline P1 & P2 & P3 & P4 & P5 & Ref. \\
\hline 8,43 & 3,88 & 3,88 & 8,43 & 3,88 & 5,95 \\
\hline \hline
\end{tabular}

Fonte: Elaborado pelos autores.

\subsection{Fase "3d" - Comparação Gráfica Relativa entre Projetos}

Os benefícios consolidados e os números de prioridade de risco são indicados em forma de coordenadas, nas quais os primeiros são localizados no eixo das ordenadas e os últimos no eixo das abcissas. São inseridos, ainda, os valores de referência. 
A representação no Gráfico 1 facilita a análise bidimensional de posicionamento dos projetos quanto ao benefício e risco.

O quadrante superior esquerdo contém os projetos que agregam valor acima da média e que representam baixo risco relativo: P2, P3 e P5. Segundo o critério de julgamento do especialista em $P \& D$, são considerados como prioritários e devem ser encaminhados para contratação e posterior execução. Nesse caso, a coordenação e o acompanhamento devem ser minuciosos para garantir que objetivos, metas e produtos pretendidos sejam atendidos conforme o esperado. Reavaliações dos projetos deverão ocorrer ao longo do cronograma até o encerramento, havendo a entrega dos produtos no modo como foram previstos.

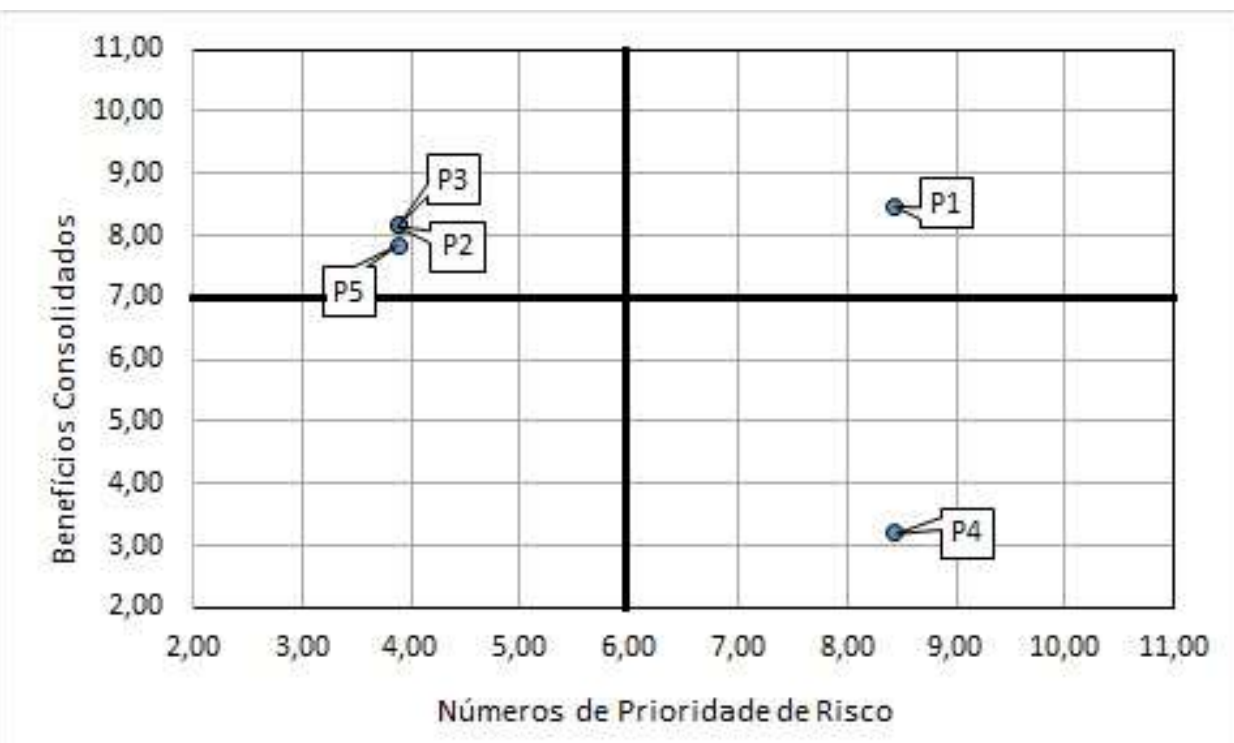

Gráfico 1 - Posicionamento dos Projetos quanto aos Benefícios e aos Riscos

Fonte: Elaborado pelos autores.

O quadrante superior esquerdo contém os projetos que agregam valor acima da média e que representam baixo risco relativo:P2, P3 e P5. Segundo o critério de julgamento do especialista em $P \& D$, são considerados como sendo prioritários e devem ser encaminhados para contratação e posterior execução. Nesse caso, a coordenação e o acompanhamento devem ser minuciosos para garantir que objetivos, metas e produtos pretendidos sejam atendidos conforme o esperado. Reavaliações dos projetos deverão ocorrer ao longo do cronograma até o encerramento, havendo a entrega dos produtos no modo como foram previstos.

$\mathrm{O}$ quadrante superior direito exibe o projeto $\mathrm{P} 1$, o qual apresenta superioridade na geração de benefícios para a empresa patrocinadora, entretanto se caracteriza por elevado risco de não conformidade. Isto quer dizer que, muito provavelmente, dois elementos poderão vir a comprometer a forte agregação de valor proposta pelo projeto: riscos de impossibilidade de atender o caráter de inovação tecnológica (não representa uma solução inovadora), ou a impraticabilidade de execução adequada com os recursos materiais, com os equipamentos e com as despesas - elementos direcionadores de risco que dominam o vetor 
de pesos. Nesse caso, caberá à empresa avaliar sua propensão a assumir tal risco, e, portanto, a intenção de levar adiante sua contratação e sua execução, o que demandará esforço adicional de coordenação e de acompanhamento.

$\mathrm{O}$ quadrante inferior direito exibe o projeto $\mathrm{P} 4$, o qual apresenta baixa proposta de agregação de benefícios e, ademais, elevado risco de não conformidade. Nesse caso, a empresa deve considerar fortemente a possibilidade de abandonar essa solução, não prosseguindo com sua contratação. Eventualmente deverá passar por uma restruturação ou ser executado em outro contexto, distinto dos projetos de P\&D.

\section{CONSIDERAÇÕES FINAIS}

Assim como em todos os processos empresariais, a busca pela racionalização encontra soluções que proporcionam resultados satisfatórios na padronização de critérios, na gestão dos objetivos, e no cuidado em se adotarem métodos transparentes para escolher projetos de P\&D. A proposta de uma metodologia para esse processo tem como objetivo tentar se distanciar da improvisação, da decisão sem explicação racional e do risco de que ocorra algo de errado. No entanto, a racionalização esbarra na complexidade, na subjetividade dos critérios, na forte influência da cultura organizacional, na especificidade das situações, na necessidade de diversificação e na conjuntura econômica e social.

A utilização da análise multicritério com lógica fuzzy se revela como um meio analítico para hierarquizar e mapear tais riscos na sistemática de avaliação e de seleção de projetos de P\&D. A proposta de um procedimento sistemático para a coleta de dados e para a execução de uma metodologia de gestão de riscos específicos para as empresas de energia elétrica preencherá essa lacuna e propiciará adotar tais iniciativas. O objetivo principal será propor tal metodologia, viabilizada por meio do julgamento de especialistas, tendo os riscos caracterizados pelo NPR para cada projeto.

Um risco é qualquer evento que pode afetar, positiva ou negativamente, os objetivos de um projeto, e uma concepção inovadora é campo fértil para riscos e incertezas. A dimensão de um projeto de P\&D está diretamente relacionada ao tamanho do impacto e à probabilidade de que um evento imprevisto possa vir a causá-lo, entretanto, não se limita apenas a isso. A complexidade, o cronograma, a forma de gestão, dentre outros, também são elementos que interferem no perfil de risco. Alguns desses eventos são incontroláveis, externos, muitos deles vinculados a mudanças regulatórias e a reorientações de variáveis macroeconômicas. Outros são parcial ou totalmente controláveis, podem ser mitigados ou até mesmo evitados, a exemplo da conformidade dos serviços desempenhados pelas instituições executoras.

Portanto, a identificação dos direcionadores de risco se concentra naqueles que podem afetar o sucesso do projeto, e busca verificar suas características. Trata-se de processo iterativo, visto que outros podem ser descobertos durante todo o ciclo de vida do projeto. Dessa forma, a ideia principal do processo de identificação é determinar e analisar, de maneira preventiva, os fatores relevantes que poderiam acontecer e que apresentariam impacto importante no alcance dos objetivos do projeto. Esse processo de identificação, 
quando bem feito, pode reduzir futuros custos e surpresas indesejadas ao longo do desenvolvimento e da implantação. Quanto mais tarde um dado risco for identificado ao longo da linha temporal do projeto, maiores serão os custos a que o empreendedor estará sujeito na implantação das medidas para sua mitigação.

A metodologia especificada foi operacionalizada em ambiente Web utilizando dll's na camada de aplicação e MS SQL Server na camada de persistência. O uso de uma linguagem compilada se mostra mais adequada que uma linguagem interpretada devido ao melhor desempenho e à complexidade da estrutura de dados envolvida na lógica fuzzy. $\mathrm{O}$ uso do banco de dados da Microsoft não gera custo, uma vez que será utilizada a versão gratuita, e não dificulta qualquer possível integração com outros bancos. O sistema SABER (Sistema de Avaliação dos Benefícios e Riscos em Projetos de P\&D) está operando nas empresas do setor elétrico Light, Cemig, Taesa e TBE desde meados de junho de 2016.

\section{Referências}

ANEEL. Manual do Programa de Pesquisa e Desenvolvimento Tecnológico do Setor de Energia Elétrica. Brasilia: ANEEL, 2012.

CHANG, D.Y. Applications of the extent analysis method on fuzzy AHP, European Journal of Operational Research, v.95, p.649-655, 1996.

FIGUEIRA, J., GRECO, S., \& EHRGOTT, M. Multiple criteria decision analysis: State of art surveys. New York: Springer, 2005.

FIGUEIRA, J.; ROY, B. Determining the Weights of Criteria in the ELECTRE Type Methods with a Revised Simos' Procedure. European Journal of Operational Research, v.139, n.2, p.317-326, 2002.

HUANG, C.C.; CHU, P.Y.; CHIANG, Y.H. A fuzzy AHP application in government-sponsored R\&D project selection. Omega, v.36, n.6, p.1038-1052, 2008.

LEUNGA, C., CAOB, D. On consistency and ranking of alternatives in fuzzy AHP. European Journal of Operational Research, v.124, p.102-113, 2000.

LIGHT. Metodologia de gestão de portfolio de projetos. Rio de Janeiro: Light, 2014.

MEIXNER, O. Fuzzy AHP Group Decision Analysis and its Application for the Evaluation of Energy Sources, Hamburg: Institute of Marketing and Innovation, 2011.

MONTIS, A. et al. Assessing the quality of different MCDA methods. In: GETZNER, M.; SPASH, C.; STAGL, S. (Org.). Alternatives for Environmental Evaluation. Abingdon: Routledge, 2005, p.99-133.

PAN, N.F. Fuzzy AHP approach for selecting the suitable bridge construction method. Automation in Construction, 17, p.958-965, 2008.

RIBAS, J.R. Modelo benefício $\mathbf{x}$ risco para a classificação de projetos de pesquisa e eficiência energética. Notas Técnicas, Rio de Janeiro: Coppetec, 2015.

SAATY, T. L. The Analytic Hierarchy Process, New York: McGraw-Hill, 1980.

SAATY, T. L. Extending the Measurement of Tangibles to Intangibles. International Journal of Information Technology and Decision Making, v.8, n.1, p.7-27, 2009.

TANG, Y.; BEYNON, M. Application and Development of a Fuzzy Analytic Hierarchy Process within a Capital Investment Study. Journal of Economics and Management, v.1, p.207-230, 2005.

TEOH, P.C.; CASE, K. Modeling and reasoning for failure modes and effects analysis generation, in Proceedings of the Institution of Mechanical Engineers - Part B - Journal of Engineering Manufacture, v.218, n.3, p.289-300, 2004. 
ZAVADSKAS, E.K.; TURSKIS, Z.; TAMOŠAITIENE, J.; MARINA, V. Multicriteria Selection of Project Managers by Applying Grey Criteria. Technological and Economic Development of Economy, p.14, n.4, p.462-477, 2008.

ZHUA, K.J., JINGA, Y.; CHANG, D.Y. A discussion on Extent Analysis Method and applications of fuzzy AHP. European Journal of Operational Research, v.116, p.450-456, 1999. 